\title{
Botox: Information versus Werbung
}

\author{
Wie können Ärztinnen und Ärzte in der ästhetischen Medizin über ärztliche \\ Behandlungen mit Botulinumtoxin (Botox) informieren, ohne dafür zu werben? \\ Gestützt auf ein Gerichtsurteil kann Swissmedic jetzt Leitlinien veröffentlichen.
}

Swissmedic - Schweizerisches Heilmittelinstitut Hallerstrasse 7

CH-3000 Bern 9

Tel. 0313220276

Fax 0313220406

odette.klensch[at]swissmedic.ch
Das Bundesverwaltungsgericht stützt einen Entscheid von Swissmedic über Verbote von Arzneimittelwerbung im Rahmen der Anpreisung von ärztlichen Dienstleistungen (vgl. rechtskräftiges Urteil vom 17. Oktober 2011; C-1795/2009 [1]). Konkret bedeutet dies Folgendes:

Ausschliesslich Informationen allgemeiner Art über die Gesundheit oder über Krankheiten sind zulässig und fallen nicht in den Geltungsbereich der heilmittelrechtlichen Werbebestimmungen - solche Informationen sind somit nur zulässig, sofern sie sich weder direkt noch indirekt auf bestimmte Arzneimittel beziehen.

Damit Webseiten im Rahmen der Bewerbung von ärztlichen Dienstleistungen den werberechtlichen Bestimmungen der Heilmittelgesetzgebung entsprechen, darf namentlich der Begriff «Botox» nicht als Kennzeichnung für spezifische Anwendungen von botulinumtoxinhaltigen Arzneimitteln benutzt werden, da deren Nennung und Abbildung als unerlaubte Werbung für verschreibungspflichtige Arzneimittel qualifiziert werden muss. Der Begriff «Botox» darf auch in keiner Weise hervorgehoben werden; dessen Verwendung als Titel oder im Titel oder als Überschrift einer Rubrik und/oder Unterrubrik sowie innerhalb des Domainnamens oder in Internetadressen (Pfad, URL) ist nicht zulässig. Erlaubt ist hingegen die Wirkstoff-Bezeichnung «Botulinumtoxin» als Überschrift von Rubriken oder die Erläuterung des Begriffs «Botox-Behandlung» innerhalb eines Fliesstextes.

Damit alle Informationen die Kriterien von Vollständigkeit, Ausgewogenheit und Sachlichkeit erfüllen, sind zusätzlich folgende Aspekte zu berücksichtigen:

\section{Zugelassene Arzneimittel und zugelassene} Indikationen des Wirkstoffes Botulinumtoxin Insgesamt sind drei Präparate mit dem Wirkstoff Botulinumtoxin zugelassen, jedoch nur eines davon für eine Behandlung, die auch ästhetisch motiviert sein kann (Behandlung von mittelschweren bis schweren Glabellafalten bei Erwachsenen, auch «Zornesfalten» genannt). Weil Indikationen, die gegenüber dem Publikum angeboten werden, im Einklang mit der Arzneimittelinformation stehen müssen, ist für ästhetische Behandlungen mit Botuli- numtoxin lediglich die Erwähnung der Behandlung bei Zornesfalten zulässig. Alle relevanten Rubriken einer Webseite, die über ästhetisch motivierte Behandlungen mit Botulinumtoxin informieren, dürfen deshalb nur diese Information enthalten sowie allenfalls auf die Behandlung von übermässigem Schwitzen in der Achselhöhle mittels Botulinumtoxin hinweisen, die ebenfalls zugelassen ist.

\section{Off Label Use}

In der Faltenbehandlung ist wie erwähnt einzig die Behandlung von Glabellafalten («Zornesfalten») eine zugelassene Indikation. Der Einsatz für das Glätten der übrigen Gesichtsfalten (z. B. Krähenfüsse, Stirnfalten, Nasenfältchen usw.) oder weitere Anwendungen (z. B. Migräne, Spannungskopfschmerzen usw.) gelten als sog. «Off Label Use», also als Anwendung eines zugelassenen Arzneimittels ausserhalb des von der Swissmedic genehmigten Gebrauchs. Dafür trägt alleine der behandelnde Arzt die vollumfängliche Verantwortung und er muss die Patientin/den Patienten entsprechend informieren.

\section{In der Faltenbehandlung ist einzig die Behandlung von Glabellafalten} \section{(«Zornesfalten») eine zugelassene}

\section{Indikation.}

Eine Bewerbung von nicht zugelassenen Indikationen ist nicht erlaubt. Hinweise auf Webseiten für solche Off-Label-Behandlungsmöglichkeiten sind deshalb grundsätzlich unzulässig. Swissmedic akzeptiert jedoch den Oberbegriff «mimisch bedingte Falten» in Fliesstexten und die Aufzählung von spezifisch zu behandelnden Falten (auch von solchen, die Off Label Use darstellen) im Rahmen von Preisangeboten, sofern dies in zurückhaltender Art und Weise geschieht.

\section{Angaben zur Sicherheit}

In der von der Swissmedic genehmigten Arzneimittelinformation über die Behandlung von Glabellafalten 
(«Zornesfalten») mittels Botulinumtoxin wird festgehalten, dass aufgrund von Untersuchungsergebnissen aus kontrollierten klinischen Studien bei der Behandlung für 24\% der Patientinnen und Patienten behandlungsbedingte unerwünschte Wirkungen zu erwarten sind. Diese verschiedenen möglichen Nebenwirkungen werden dort klassifiziert nach der Wahrscheinlichkeit ihres Eintritts: sehr häufig (>10\%), häufig (1\%-10\%) und gelegentlich $(0,1 \%-1 \%)$.

Alle relevanten Rubriken einer Webseite müssen nicht nur hinsichtlich der möglichen Anwendungen (Indikationen), sondern auch bezüglich ihrer Angaben zur Sicherheit mit der genehmigten Arzneimittelinformation übereinstimmen. Swissmedic empfiehlt daher neben einer, evtl. zusammenfassenden, Aussage zu den Risiken einer Behandlung die Aufschaltung eines entsprechenden Links zur Datenbank mit den genehmigten Arzneimittelinformationen.

\section{Tabelle 1}

Zulässige Angaben im Rahmen der Anpreisung von ärztlichen Dienstleistungen (Behandlungen mit Botulinumtoxin).

\begin{tabular}{|c|c|}
\hline Indikationen & $\begin{array}{l}\text { Glabellafalte (Zornesfalte) } \\
\text { mimisch bedingte Falten (ohne spezifische Aufzählung) } \\
\text { übermässiges Schwitzen in den Achselhöhlen }\end{array}$ \\
\hline Nebenwirkungen & $\begin{array}{l}\text { Als sachliche und ausgewogene Information zwingend } \\
\text { erforderlich, jedoch nur Angaben basierend auf der von } \\
\text { Swissmedic genehmigten Arzneimittelinformation (Fach-) }\end{array}$ \\
\hline Wirkungseintritt, -dauer & $\begin{array}{l}\text { Nur Angaben basierend auf der von Swissmedic genehmigten } \\
\text { Arzneimittelinformation (Fach-) }\end{array}$ \\
\hline Botulinumtoxin & Angabe des Wirkstoffes \\
\hline «Botox»-Behandlungen & $\begin{array}{l}\text { Begriff «Botox» nur als Beschreibung innerhalb eines } \\
\text { Fliesstextes }\end{array}$ \\
\hline Fachinformation & Aufschaltung \\
\hline
\end{tabular}

Tabelle 2

Nicht zulässige Angaben im Rahmen der Anpreisung von ärztlichen Dienstleistungen (Behandlungen mit Botulinumtoxin).

\begin{tabular}{|c|c|}
\hline $\begin{array}{l}\text { Bezeichnung oder Abbildung } \\
\text { von Botulinumtoxin-haltigen } \\
\text { Arzneimitteln }\end{array}$ & Im gesamten Text \\
\hline Begriff «Botox» & $\begin{array}{l}\text { Innerhalb Domainname } \\
\text { auf der Homepage von Webseiten } \\
\text { als Titel einer Rubrik } \\
\text { als Titel einer Unterrubrik } \\
\text { innerhalb Internetadressen (Pfad, URL) }\end{array}$ \\
\hline Off Label Use & $\begin{array}{l}\text { Angaben, wie z.B. Krähenfüsse, Stirnfalten, Nasenfältchen, } \\
\text { Lippenfalten, Halsfalten, Migräne usw. }\end{array}$ \\
\hline Vorher-Nachher-Bilder & Einstufung als garantierte Wirkversprechen \\
\hline $\begin{array}{l}\text { Spezialaktionen, } \\
\text { die sich auf den Preis } \\
\text { der Behandlungen auswirken }\end{array}$ & Förderung eines übermässigen Einsatzes \\
\hline
\end{tabular}

\section{Angaben zum Wirkungseintritt und zur Wirkungsdauer}

Ebenso müssen alle relevanten Rubriken einer Webseite bezüglich Angaben zum Wirkungseintritt und zur Wirkungsdauer mit der genehmigten Arzneimittelinformation übereinstimmen.

\section{Vorher-Nachher-Bilder}

Abbildungen und Fotografien, die den Zustand vor der Behandlung und denjenigen nach der Behandlung dokumentieren, müssen als klassische Darstellungen von garantierten Wirkversprechen der angewendeten Arzneimittel gewertet werden und sind nicht zulässig. Sie wären selbst dann unzulässig, wenn die Botulinumtoxin-Präparate nicht verschreibungspflichtig wären. Vorher-Nachher-Bilder dürfen in keinem Fall auf Webseiten enthalten sein.

\section{Angebot von Spezialaktionen, die sich auf den Preis der Behandlung mit Botulinum- toxin auswirken}

Als besonders gravierende Verstösse sind Spezialaktionen anzusehen, bei denen für Behandlungen mit Botulinumtoxin Rabatte angeboten werden. Neben der Verletzung des Publikumswerbeverbots wird auf diese Weise auch Arzneimittelwerbung betrieben, die zu einem übermässigen, missbräuchlichen oder unzweckmässigen Einsatz verleiten und damit die Gesundheit der Behandelten gefährden kann.

Spezialaktionen für ästhetische Behandlungen mit Botulinumtoxin sind daher verboten und werden durch Swissmedic strafrechtlich verfolgt.

\section{Referenzen}

1 www.bvger.ch/publiws/pub/search.jsf $\rightarrow$ unter Suchtext C-1795/2009 eingeben und Suchen

- Heilmittelgesetz (HMG): Bundesgesetz vom 15. Dezember 2000 über Arzneimittel und Medizinprodukte (Heilmittelgesetz, HMG; SR 812.21) www.admin.ch/ch/d/sr/c812_21.html

- Botschaft zu HMG: www.amtsdruckschriften.bar. admin.ch $\rightarrow$ Suchbegriff HMG eingeben oder direkt: www.amtsdruckschriften.bar.admin.ch/viewOrigDoc. do?id=10055092

- Arzneimittel-Werbeverordnung (AWV): Verordnung vom 17. Oktober 2001 über die Arzneimittelwerbung (Arzneimittel-Werbeverordnung, AWV; SR 812.212.5) www.admin.ch/ch/d/sr/c812_212_5.html

- Medizinal-Berufegesetz (MedBG): Bundesgesetz vom 23. Juni 2006 über die universitären Medizinalberufe (Medizinalberufegesetz, MedBG; SR 811.11) www. admin.ch/ch/d/sr/c811_11.html

- Bundesverwaltungsgericht; Urteil C-1795/2009 www.bvger.ch/publiws/pub/search.jsf $\rightarrow$ unter Suchtext C-1795/2009 eingeben und Suchen 